\title{
Theory and Applications of Focal-Series Reconstruction in HRTEM
}

\author{
Andreas Thust
}

Ernst Ruska-Centre for Microscopy and Spectroscopy with Electrons (ER-C) and Institute of Solid State Research, Forschungszentrum Jülich GmbH, D-52425 Jülich, Germany

The interpretation of high-resolution electron microscopy (HRTEM) images is usually not a trivial task due to two major physical processes: First, the diffraction of an initially plane electron wave falling on the object is governed by quantum-mechanical principles, resulting in a mathematically complex-valued wave function at the exit-plane of the object (exit-plane wave function, EPW), which consists of an amplitude and a phase part. Second, the EPW is further modified upon its transfer through the lens system of the microscope before its actual imaging on a detector. Lens aberrations such as spherical aberration and defocus distort the EPW, leading to a strong blurring of the images. Finally the phase of the wave function is lost upon imaging, meaning an unwanted information loss.

The information modification and the information loss caused by the imaging process can be overcome by retrieving the EPW. The EPW is ideally free of all imaging artifacts caused by the observation instrument and allows in many cases a straightforward structure interpretation, especially when using very thin samples on the order of a few nanometers, since then the phase of the EPW reveals atomic columns quite directly (see Fig. 1). In order to have the desired EPW available, an inversion of the imaging process can be achieved by numerical procedures, which use as input typically 10-20 images taken at different values of the objective lens defocus [1-5]. The core principle behind the retrieval of the EPW from a focal series lies in the fact that the EPW is the generating function behind all images and that each image contains a different portion of the EPW, albeit in a highly encoded form. The aim of reconstruction schemes is to re-assemble the EPW from the information dispersed over the whole focal series.

Having the wave function available, it is possible to eliminate not only defocus and spherical aberration numerically, but also all further unwanted residual lens aberrations like coma, two- and threefold astigmatism [6]. In combination with the high signal-to-noise ratio of the EPW compared to a single image, it is possible to perform high-precision measurements of atomic positions [7] and to detect columns of light elements such as $\mathrm{C}, \mathrm{N}$, and $\mathrm{O}$ with sub Angstrom resolution [8]. Recent experiments demonstrate the usefulness of focal-series reconstruction when applied to images made with an electron microscope corrected for spherical aberration [9].

\section{References}

[1] P. Schiske, Proc. Fourth Regional Congr. on Electron Microscopy, Rome, 1968, Vol. 1, p. 145.

[2] W.O. Saxton, in: Advances in Electronics and Electron Physics, Academic Press, London, 1978.

[3] E.J. Kirkland, Ultramicroscopy 15 (1984) 151.

[4] W. Coene et al., Ultramicroscopy 64 (1996) 109.

[5] A. Thust et al., Ultramicroscopy 64 (1996) 211.

[6] A. Thust et al., Ultramicroscopy 64 (1996) 249.

[7] C.L. Jia and A. Thust, Phys. Rev. Lett. 82 (1999) 5052.

[8] C. Kisielowski et al., Ultramicroscopy 89 (2001) 243.

[9] K. Tillmann, A. Thust and K. Urban, Microsc. Microanal. 10 (2004) 185. 

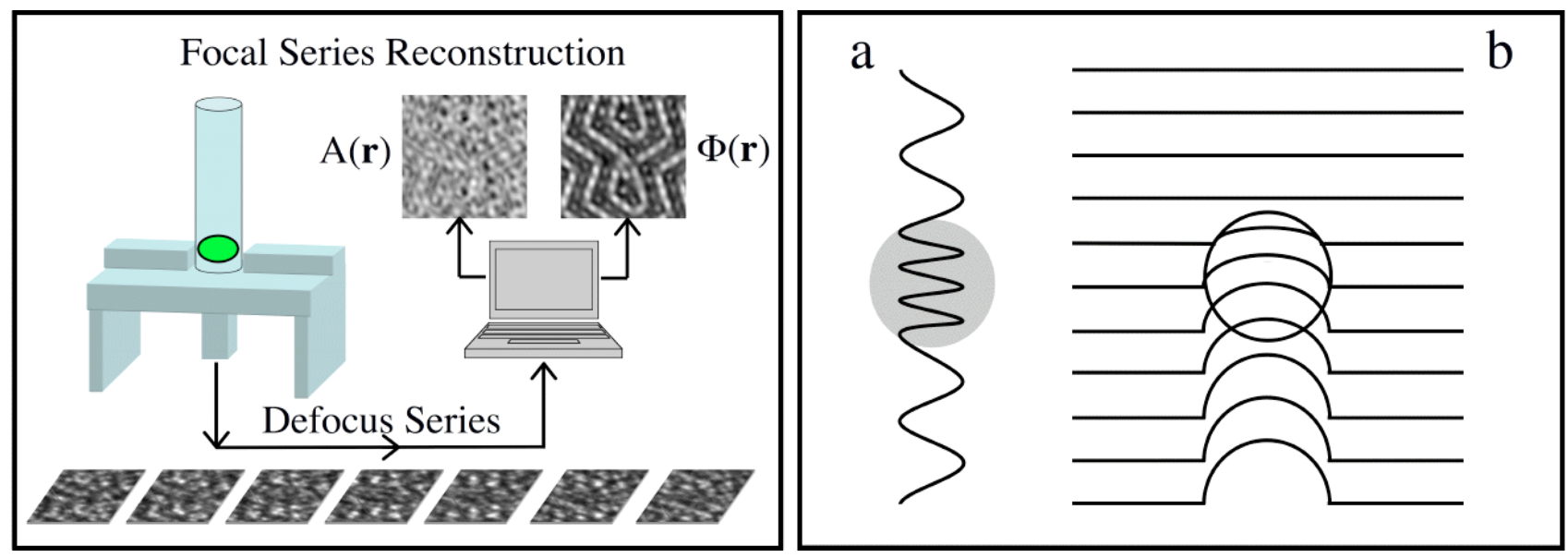

FIG. 1. Left: Principle of focal-series reconstruction in HRTEM: A series of typically 10-20 high resolution images is recorded from the same object area with different values of the objective lens defocus. The images are then transferred to a computer, which retrieves the quantum-mechanical electron wave function at the exit-plane of the object. The resulting wave function is complex-valued and is described by an amplitude $\mathrm{A}(\boldsymbol{r})$ and a phase $\Phi(\boldsymbol{r})$. Right: Schematic explanation of the behavior of the phase $\Phi(\boldsymbol{r})$ on traversal of an atom: (a) Since the atom constitutes an optically denser medium than the surrounding vacuum, the electron wavelength is shortened. (b) Lines of equal phase resulting from the shortening of the electron wavelength. The fallback of the iso-phase lines at atomic positions means that the phase of the wave function is peaked there.
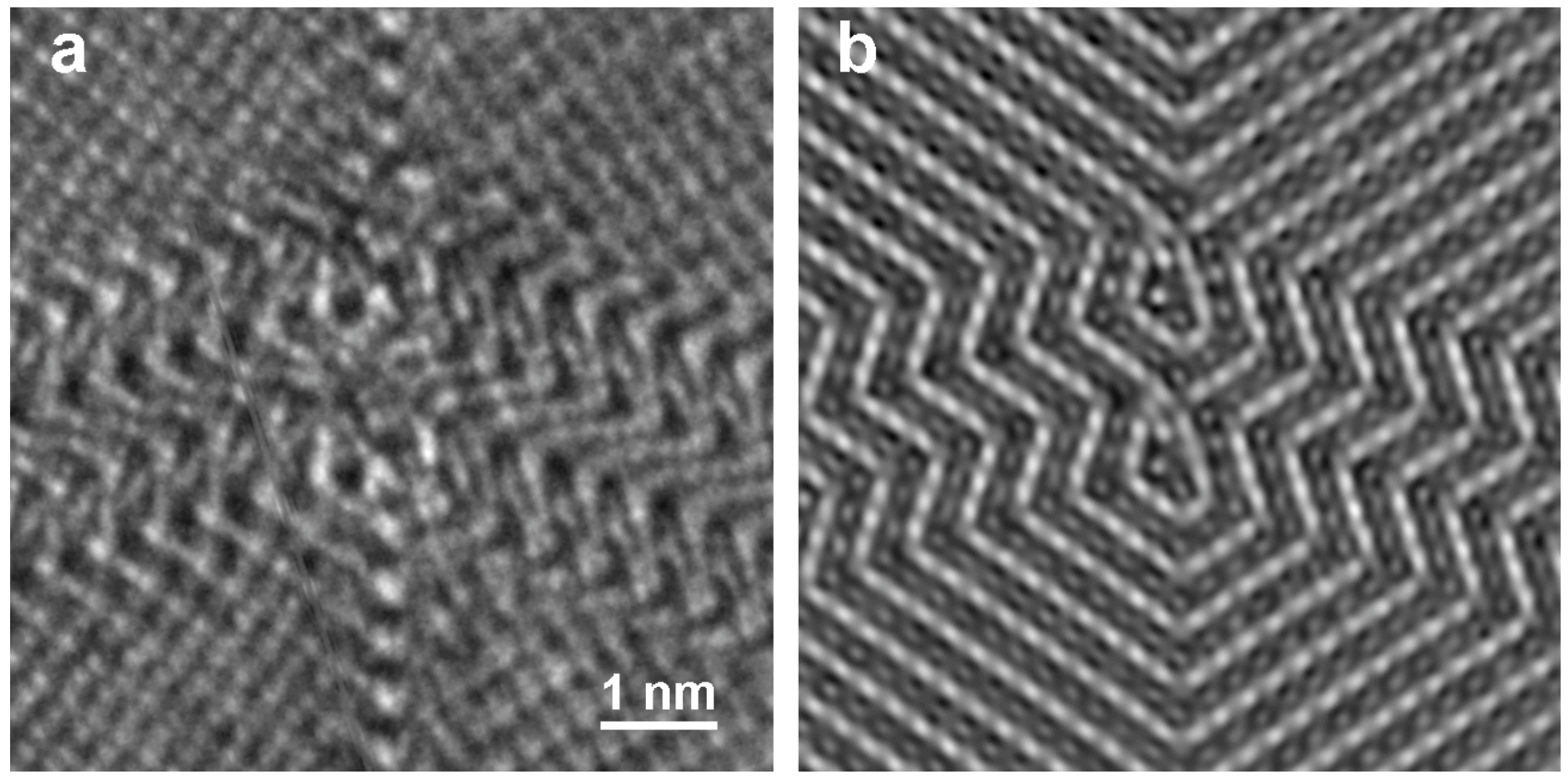

FIG. 2. (a) High-resolution image representative for a focal series of 20 images recorded with a Philips CM20 FEG electron microscope. The object is multiply twinned $\mathrm{BaTiO}_{3}$ viewed along the [110] zone axis. The image is blurred due to unwanted optical aberrations, mainly due to spherical aberration and defocus. (b) Phase of the reconstructed and aberration-free wave function: For a thin object the phase peaks are located at atomic columns and enable thus a direct structure interpretation. 